\title{
Estrutura da vegetação colonizadora em ambiente degradado por extração de cascalho em Diamantina, MG
}

\author{
Israel Marinho Pereira ${ }^{1}$, Anne Priscila Dias Gonzaga ${ }^{2 \star}$, Evandro Luiz Mendonça Machado¹, Marcio Leles Romarco Oliveira, \\ Izabel Cristina Marques ${ }^{1}$ \\ ${ }^{1}$ Universidade Federal dos Vales do Jequitinhonha e Mucuri, Departamento de Engenharia Florestal, Rodovia MGT 367, Km 583, n 5000, Alto da Jacuba, \\ CEP 39100-000, Diamantina, MG, Brasil \\ ${ }^{2}$ Universidade Federal dos Vales do Jequitinhonha e Mucuri, Curso de Geografia, Rodovia MGT 367, Km 583, n 5000, Alto da Jacuba, CEP 39100-000, Diamantina, \\ MG, Brasil
}

\author{
"Autor correspondente: \\ diaspri@gmail.com \\ Termos para indexação: \\ Adaptações ambientais \\ Histórico de degradação \\ Sucessão ecológica \\ Index terms: \\ Environmental adaptations \\ History of degradation \\ Ecological succession
}

Histórico do artigo:

Recebido em 14/08/2014

Aprovado em 23/04/2015

Publicado em 30/06/2015

doi: $10.4336 / 2015 . p f b .35 .82 .769$
Resumo - Este trabalho procurou identificar e quantificar espécies colonizadoras e estrutura em área de empréstimo, assim como listar espécies para recuperação de áreas degradadas. Foram selecionados três ambientes, sendo o ambiente 1 (A1) com histórico de uso menos intenso; ambiente 2 (A2) nível de degradação intermediário e ambiente 3 (A3) com maior degradação. Nos três ambientes foram sorteadas 20 parcelas de $25 \mathrm{~m}^{2}$. Nestas, foram amostrados todos os indivíduos com altura $\geq 0,10 \mathrm{~m}$ e DAS $\leq 5,0 \mathrm{~cm}$. Foram calculados os índices de diversidade de Shannon (H'), equabilidade de Pielou (J'), similaridade de Jaccard e Czekanowski, assim como os parâmetros fitossiológicos e a análise de espécies indicadoras. Foram registradas 32 espécies pertencentes a 15 famílias. O ambiente A1 foi o mais rico em espécies e com maior densidade e A2 apresentou a menor riqueza e densidade. Os ambientes apresentaram baixa similaridade florística e estrutural, sendo observadas diferenças estatísticas para a densidade (sendo encontrados 844, 270 e 497 indivíduos em A1, A2 e A3, respectivamente) e diversidade que variou entre 1,680 a 2,074 nats.indivíduo ${ }^{-1}$. Provavelmente, estas divergências se devem às variações do ambiente impostas pelas diferentes intensidades de degradação.

\section{Colonizer vegetation structure in gravel mining degraded environment in Diamantina, MG, Brazil}

\begin{abstract}
This work aimed at identifying and quantifying colonizer species and structure in gravel mining degraded environment and to identify species for restoration. It was selected three sites characterized as: site 1 (S1), place with less intense history of use; site 2 (S2), intermediary level of degradation; and site 3 (S3), with higher degradation. Within the three sites it was withdrawn 20 plots of $25 \mathrm{~m}^{2}$. Within each plot, species with height $\geq 0.10 \mathrm{~m}$ and DNS $\leq 5.0 \mathrm{~cm}$ were sampled. It was calculated Shannon's diversity (H'), Pielou's equability (J'), Jaccard's and Czekanowski's similarity indices, as well as the phytosociology parameters, besides the analysis of indicator species. Thirty-two species and 15 families were registered. S1 was the most species-rich, with the highest density, while S2 presented the lowest richness and density. The sites showed low floristic and structural similarity and statistical differences regarding density, (it was found 844, 270 and 497 individuals in, sites A1, A2 and A3, respectively). Diversity varied from 1,680 to 2,074 nats.individual ${ }^{-1}$. Probably, these divergences are due to the environment variations imposed by the different degradation intensities.
\end{abstract}




\section{Introdução}

Dentre as atividades humanas mais impactantes, a mineração se destaca na contribuição de forma significativa para a degradação do meio físico desde escalas pontuais até regionais (Gomes et al., 2000; Cooke \& Johnson, 2002). As atividades minerárias podem ser desenvolvidas de várias formas, sendo a área de empréstimo definida como um local onde foram retiradas a cobertura vegetacional original e a camada superficial do solo, geralmente expondo o horizonte $\mathrm{C}$ e causando compactação, perda de matéria orgânica e baixa disponibilidade de nutrientes do solo, o que acarreta prejuízos aos mananciais e à fauna local (Ferreira et al., 2007).

Assim, faz-se necessária a restauração destes ambientes, sendo esta iniciada com a criação de condições que impulsionem os caminhos da sucessão (Anand \& Desrochers, 2004). A aceleração da recuperação de áreas degradadas pode ser obtida pelo plantio de espécies (Parrotta et al., 1997). Entretanto, estudos mais detalhados que visem aperfeiçoar estas técnicas de plantio são necessários, principalmente quando se tratam de espécies nativas em áreas mineradas no Cerrado (Silva \& Corrêa, 2008).

De acordo com Nappo et al. (2000), dentre os principais aspectos que devem ser considerados na recuperação de áreas mineradas, tem-se a revegetação, que envolve seleção das espécies, combinação de grupos ecológicos e arranjo do plantio; condução da regeneração natural e plantios de enriquecimento. Desta forma, estudos básicos como composição florística e estrutura da comunidade recolonizadora de áreas de mineração abandonadas devem ser considerados ferramentas importantes a serem empregadas nas estratégias para recuperação de áreas mineradas (Parrota et al., 1997; Klein et al., 2009), possibilitando a seleção de espécies mais adaptadas às condições locais e, consequentemente, com maior chance de sucesso no plantio (Bentes-Gama et al., 2008).

Este trabalho teve como objetivo identificar e quantificar as espécies que colonizaram espontaneamente ambientes de área de empréstimo no Parque Estadual do Biribiri, assim como comparar a flora e estrutura destes ambientes e listar espécies com potencial para a recuperação de áreas degradadas pela mineração.

Pesq. flor. bras., Colombo, v. 35, n. 82, p. 77-88, abr./jun. 2015

\section{Material e métodos}

\section{Localização e caracterização da área de estudo}

Para a realização deste estudo selecionou-se dentro do Parque Estadual do Biribiri uma área de empréstimo que teve origem com a retirada de cascalho em meados de 1950 com a construção da rodovia BR-367 que liga Belo Horizonte a Diamantina (Borsagli, 2010). O processo de recolonização dessa área está ocorrendo de maneira mais efetiva desde 1998, com a criação do Parque Estadual do Biribiri (PEB), quando cessou a extração de cascalho.

A área da cascalheira situa-se entre as coordenadas $18^{\circ} 11^{\prime} 54,4^{\prime \prime} \mathrm{S}$ e $43^{\circ} 35^{\prime} 05,9^{\prime \prime} \mathrm{W}$ e $18^{\circ} 11^{\prime} 59,8^{\prime}$ " S e $43^{\circ} 35^{\prime} 10,2^{\prime \prime}$ W e altitude média de $1.412 \mathrm{~m}$, compreende aproximadamente 10 ha e se encontra em diferentes processos de recuperação.

O clima na região é do tipo $\mathrm{Cwb}$, de Köppen (mesotérmico com verões brandos e suaves e estiagens de inverno). $\mathrm{O}$ clima na região apresenta temperaturas médias entre $18{ }^{\circ} \mathrm{C}$ e $20{ }^{\circ} \mathrm{C}$, sendo registradas temperaturas mais elevadas em dezembro e janeiro e temperaturas mais baixas em junho e julho. No período chuvoso a média de precipitação é de $223 \mathrm{~mm}$, sendo a média anual de 1.351,22 mm (Neves et al., 2005). Fisionomicamente o PEB caracteriza-se pelo predomínio de formações savânicas (cerrados sentido restrito e rupestre), campestres (campos limpos, úmidos e rupestres) e florestais (florestas estacionais semideciduais), constituindo um ecótone entre o Cerrado e a Mata Atlântica. Contudo, é marcante a presença de áreas em diferentes estágios de regeneração pósdistúrbio.

A vegetação predominante no entorno da área de estudo é o cerrado stricto sensu, e manchas de floresta estacional semidecidual. Os solos predominantes na área antes da extração de cascalho eram caracterizados como Plintossolos, constituídos por material mineral, com horizonte plíntico ou litoplíntico ou concrecionário iniciando dentro de $40 \mathrm{~cm}$, ou dentro de $200 \mathrm{~cm}$ quando imediatamente abaixo do horizonte A ou E, ou de outro horizonte que apresente cores pálidas, variegadas ou com mosqueados em quantidade abundante (Santos et al., 2006).

Foram selecionados em uma cascalheira (área de empréstimo), três ambientes distintos em função dos níveis de degradação e cobertura de vegetação, conforme caracterizados na tabela 1 . 
Tabela 1. Caracterização de três sítios em uma área de cascalheira no Parque Estadual do Biribiri, em Diamantina, MG.

\begin{tabular}{|c|c|c|c|}
\hline \multirow{2}{*}{ Variáveis } & \multicolumn{3}{|c|}{ Sítios } \\
\hline & Ambiente 1 (A1) & Ambiente 2 (A2) & Ambiente 3 (A3) \\
\hline Cota média (m) & $1.426,7$ & $1.426,96$ & $1.421,48$ \\
\hline Desnível médio (\%) & 13 & 23 & 2 \\
\hline Latitude & $18^{\circ} 12^{\prime} 5.06^{\prime \prime} \mathrm{S}$ & $18^{\circ} 12^{\prime} 5.04^{\prime \prime} \mathrm{S}$ & $18^{\circ} 11^{\prime} 57.02 ” S$ \\
\hline Longitude & $43^{\circ} 35^{\prime} 10.25^{\prime \prime} \mathrm{O}$ & $43^{\circ} 35^{\prime} 7.22^{\prime \prime} \mathrm{O}$ & $43^{\circ} 35^{\prime} 7.67^{\prime \prime} \mathrm{O}$ \\
\hline Nível de degradação & Menor & Maior & Intermediário \\
\hline $\begin{array}{l}\text { Proporção de concreções lateríticas } \\
(\%)\end{array}$ & 100 & 0 & 20 \\
\hline Proporção de pilhas de estéril (\%) & 0 & 40 & 40 \\
\hline $\begin{array}{l}\text { Proporção de rocha exposta ou } \\
\text { matacões (\%) }\end{array}$ & 0 & 60 & 40 \\
\hline Nível de intervenção & Retirada do horizonte $\mathrm{O}$ e A & Retirada até o Horizonte C & Retirada dos Horizontes A e B \\
\hline Presença de erosão & Pontual & Por todo sítio & Pontual \\
\hline $\begin{array}{l}\text { Resistência mecânica à penetração } \\
\text { (Mpa) }\end{array}$ & 2,52 & 4,5 & 3,23 \\
\hline Matriz de entorno & $\begin{array}{l}\text { Estrada de acesso ao Parque } \\
\text { (distante } 5 \mathrm{~m} \text { ) }\end{array}$ & $\begin{array}{l}\text { Sítios da cascalheira com ausência de } \\
\text { vegetação colonizadora }\end{array}$ & $\begin{array}{c}\text { Sítios da cascalheira com } \\
\text { ausência de vegetação } \\
\text { colonizadora }\end{array}$ \\
\hline $\begin{array}{l}\text { Remanescente de vegetação mais } \\
\text { próximo }\end{array}$ & $\begin{array}{c}50 \text { m de vegetação secundária } \\
\text { de Floresta Estacional } \\
\text { Semidecídua }\end{array}$ & $\begin{array}{l}200 \mathrm{~m} \text { de área de cerrado com } \\
\text { monodominância de candeia } \\
\text { (Eremanthus } \mathrm{sp} .)\end{array}$ & $\begin{array}{l}20 \mathrm{~m} \text { de área de cerrado com } \\
\text { monodominância de candeia } \\
\text { (Eremanthus } \mathrm{sp} \text {.) }\end{array}$ \\
\hline Nível de colonização & Alto & Baixo & Médio \\
\hline Tamanho do sítio em hectare & 1,2 & 0,8 & 1 \\
\hline
\end{tabular}

\section{Amostragem da vegetação colonizadora}

Em cada ambiente estudado foi plotado um bloco de $1.000 \mathrm{~m}^{2}(20 \mathrm{~m} \times 50 \mathrm{~m})$, subdividido em 40 subparcelas de $25 \mathrm{~m}^{2}(5 \mathrm{~m} \times 5 \mathrm{~m})$. Para a amostragem dos ambientes em cada um destes blocos, foram sorteadas 20 parcelas, totalizando uma área amostral por ambiente de $500 \mathrm{~m}^{2}$.

Nas parcelas foram amostrados durante o período de novembro de 2007 a janeiro de 2008 todos os indivíduos vivos com altura $\geq 0,10 \mathrm{~m}$ e diâmetro ao nível do solo $(D A S) \leq 5,0 \mathrm{~cm}$. Estes tiveram sua altura total e $D A S$ mensurados. Foram coletadas amostras botânicas de todas as espécies inventariadas, sendo o material herborizado e depositado no Herbário Dendrológico Jeanine Felfili (HDJF) da UFVJM. As espécies foram identificadas em famílias, de acordo com o sistema Angiosperm Phylogeny Group III (Angiosperm Phylogeny Group, 2009).

\section{Análise de dados}

Para cada ambiente avaliaram-se a riqueza, a diversidade e a equabilidade de espécies por meio dos índices de diversidade de Shannon (H') e equabilidade de Pielou (J') (Brower \& Zar, 1984). Realizou-se uma comparação da riqueza de espécies entre os ambientes por meio dos estimadores 'jackknife' de primeira e segunda ordem (Heltsche \& Forrester, 1983). Os valores de H' dos ambientes foram comparados pelo teste $t$ de Hutcheson $\alpha=0,05$ (Zar, 1996). Utilizou-se também a Análise de Espécies Indicadoras (Dufrêne \& Legendre, 1997) processada pelo programa PC-Ord versão 6 (McCune \& Mefford, 2011), como ferramenta para a determinação das preferências das espécies em relação aos ambientes.

A semelhança florística entre os ambientes foi verificada por meio de diagramas de Venn. Também foram calculados índices de similaridade qualitativos (Jaccard) e quantitativos (Czekanowski) (Kent \& Coker, 1992). As diferenças de densidade e riqueza por parcela foram avaliadas pelo teste de Kruskal-Wallis (P5\%). Em caso de diferença significativa, foram realizados testes de Dunn (P 5\%) (Zar, 1996).

Os padrões estruturais da comunidade regenerante foram calculados, por espécie, segundo parâmetros descritos por Müeller-Dombois \& Ellenberg (2002), a saber: densidade relativa (DRi) e frequência relativa (FR) da i-ésima espécie. Além disso, calculou-se a 
distribuição de frequências dos indivíduos por classe de diâmetro e de altura da comunidade, aplicando-se intervalos constantes.

\section{Resultados e discussão}

Quando analisado em conjunto os três ambientes estudados na cascalheira, foram registradas 32 espécies pertencentes a 15 famílias botânicas, sendo que cinco espécies foram identificadas apenas ao nível de gênero. Das 32 espécies, 11 são de hábito arbóreo, 16 arbustivo e 2 erva/herbáceo (Tabela 2). Os ambientes podem ser considerados com baixa riqueza florística, quando comparados com outros estudos realizados na região (Kamino et al., 2008).

Tabela 2. Espécies amostradas em três ambientes de uma Cascalheira localizada no Parque Estadual do Biribiri no município de Diamantina (MG), com seus respectivos parâmetros estruturais.

\begin{tabular}{|c|c|c|c|c|c|c|c|c|c|c|}
\hline \multirow{2}{*}{$\begin{array}{l}\text { Família } \\
\text { Espécies }\end{array}$} & \multirow{2}{*}{$\mathbf{H}$} & \multicolumn{3}{|c|}{$\mathrm{Ni}$} & \multicolumn{3}{|c|}{$D R$} & \multicolumn{3}{|c|}{$F R$} \\
\hline & & A1 & A2 & A3 & A1 & A2 & $\mathbf{A 3}$ & A1 & A2 & A3 \\
\hline \multicolumn{11}{|l|}{ ASTERACEAE } \\
\hline Aspilia foliosa (Gardner) Baker & E & 11 & - & - & 1,3 & - & - & 1,7 & - & - \\
\hline Baccharis elliptica Gardner & $\mathrm{AB}$ & 167 & 52 & 211 & 19,8 & 19,3 & 42,5 & 16,1 & 16,0 & 23,8 \\
\hline Eremanthus incanus (Less.) Less. & AV & 153 & 79 & 30 & 18,1 & 29,3 & 6,0 & 16,1 & 21,3 & 15,5 \\
\hline Eremanthus erythropappus (DC.) MacLeish & AV & 140 & 42 & 42 & 16,6 & 15,6 & 8,5 & 14,4 & 13,3 & 17,9 \\
\hline $\begin{array}{l}\text { Pseudobrickellia angustissima (Spreng. ex Baker) } \\
\text { R.M.King \& H.Rob. }\end{array}$ & $\mathrm{AB}$ & - & 1 & - & - & 0,4 & - & - & 1,3 & - \\
\hline \multicolumn{11}{|l|}{ BIGNONIACEAE } \\
\hline Jacaranda caroba (Vell.) A.DC. & $\mathrm{AV}$ & & 2 & & & 0,7 & & & 2,7 & \\
\hline \multicolumn{11}{|l|}{ CHRYSOBALANACEAE } \\
\hline Hirtella glandulosa Spreng. & AV & 8 & & & 0,9 & & & 3,4 & & \\
\hline \multicolumn{11}{|l|}{ ERYTHROXYLACEAE } \\
\hline Erythroxylum cuneifolium (Mart.) O.E.Schulz & $\mathrm{AB}$ & 22 & & & 2,6 & & & 5,9 & & \\
\hline Erythroxylum deciduum A.St.-Hil. & $\mathrm{AB}$ & & & 1 & & & 0,2 & & & 1,2 \\
\hline \multicolumn{11}{|l|}{ FABACEAE } \\
\hline Chamaecrista desvauxii (Collad.) Killip & & 9 & & 1 & 1,1 & & 0,2 & 1,7 & & 1,2 \\
\hline Chamaecrista sp. & $\mathrm{AB}$ & 3 & 1 & & 0,4 & 0,4 & & 0,8 & 1,3 & \\
\hline Dalbergia miscolobium Benth. & $\mathrm{AV}$ & & & 1 & & & 0,2 & & & 1,2 \\
\hline Calliandra sp & & 3 & 2 & 57 & 0,4 & 0,7 & 11,5 & 2,5 & 1,3 & 10,7 \\
\hline Mimosa cf. somnians Humb. \& Bonpl. ex Willd & AV & 3 & & & 0,4 & & & 0,8 & & \\
\hline \multicolumn{11}{|l|}{ INDETERMINADA } \\
\hline Coletada & & 1 & & & 0,1 & & & 0,8 & & \\
\hline \multicolumn{11}{|l|}{ LAMIACEAE } \\
\hline Cunila $\mathrm{Sp}$ & $\mathrm{AB}$ & 141 & 54 & 23 & 16,7 & 20,0 & 4,6 & 4,2 & 16,0 & 4,8 \\
\hline \multicolumn{11}{|l|}{ LAMIACEAE (continuação) } \\
\hline Hyptis crinita Benth. & $\mathrm{AB}$ & 30 & 4 & - & 3,6 & 1,5 & - & 11,9 & 4,0 & - \\
\hline \multicolumn{11}{|l|}{ LYTHRACEAE } \\
\hline Cuphea campestris Koehne & HB & - & - & 112 & - & - & 22,5 & - & - & 7,1 \\
\hline \multicolumn{11}{|l|}{ MALPIGHIACEAE } \\
\hline Banisteriopsis stellaris (Griseb.) B.Gates & $\mathrm{AB}$ & - & - & 1 & - & - & 0,2 & - & - & 1,2 \\
\hline Byrsonima coccolobifolia Kunth & $\mathrm{AV}$ & 2 & - & - & 0,2 & - & - & 0,8 & - & - \\
\hline Heteropterys byrsonimifolia A.Juss. & AV & - & - & 1 & - & - & 0,2 & - & - & 1,2 \\
\hline
\end{tabular}


Tabela 2. Continuação.

\begin{tabular}{|c|c|c|c|c|c|c|c|c|c|c|}
\hline \multirow{2}{*}{$\begin{array}{l}\text { Família } \\
\text { Espécies } \\
\end{array}$} & \multirow{2}{*}{$\mathbf{H}$} & \multicolumn{3}{|c|}{$\mathrm{Ni}$} & \multicolumn{3}{|c|}{$D R$} & \multicolumn{3}{|c|}{$F R$} \\
\hline & & A1 & A2 & $\mathbf{A 3}$ & A1 & $\mathbf{A 2}$ & A3 & A1 & A2 & A3 \\
\hline \multicolumn{11}{|l|}{ MELASTOMATACEAE } \\
\hline Lavoisiera pectinata Cogn & $\mathrm{AB}$ & 1 & - & - & 0,1 & - & - & 0,8 & - & - \\
\hline Lavoisiera phyllocalycina Cogn & $\mathrm{AB}$ & - & 2 & - & - & 0,7 & - & - & 2,7 & - \\
\hline Rhynchanthera sp. & $\mathrm{AB}$ & 1 & - & - & 0,1 & - & - & 0,8 & - & - \\
\hline Tibouchina candolleana (Mart. ex DC.) Cogn. & $\mathrm{AV}$ & 133 & 20 & 12 & 15,8 & 7,4 & 2,4 & 9,3 & 9,3 & 8,3 \\
\hline Trembleya cf. parviflora (D.Don) Cogn. & $\mathrm{AB}$ & 10 & 3 & 1 & 1,2 & 1,1 & 0,2 & 3,4 & 4,0 & 1,2 \\
\hline \multicolumn{11}{|l|}{ MYRSINACEAE } \\
\hline Myrsine guianensis (Aubl.) Kuntze & $\mathrm{AV}$ & - & - & 2 & - & - & 0,4 & - & - & 2,4 \\
\hline \multicolumn{11}{|l|}{ MYRTACEAE } \\
\hline Campomanesia espiritosantensis Landrum & AV & - & - & 1 & - & - & 0,2 & - & - & 1,2 \\
\hline Eugenia cf. bimarginata DC. & $\mathrm{AB}$ & 3 & - & 1 & 0,4 & - & 0,2 & 2,5 & - & 1,2 \\
\hline \multicolumn{11}{|l|}{ RUBIACEAE } \\
\hline Palicourea rigida Kunth & $\mathrm{AB}$ & 2 & - & - & 0,2 & - & - & 0,8 & - & - \\
\hline \multicolumn{11}{|l|}{ SOLANACEAE } \\
\hline Solanum cf. grandiflorum Desf. & $\mathrm{AB}$ & - & 8 & - & - & 3,0 & - & - & 6,7 & - \\
\hline \multicolumn{11}{|l|}{ VERBENACEAE } \\
\hline Stachytarpheta $\mathrm{sp}$ & $\mathrm{AB}$ & 1 & - & - & 0,1 & - & - & 0,8 & - & - \\
\hline Total & & 844 & 270 & 497 & 100 & 100 & 100 & 100 & 100 & 100 \\
\hline
\end{tabular}

A análise conjunta da composição florística em nível de família, para todas as áreas mostrou que Fabaceae e Melastomataceae apresentaram a maior riqueza (5 espécies cada), seguida por Asteraceae (4) e Malpighiaceae (3), as quais juntas representam mais de $53,12 \%$ da riqueza em espécies registradas. No entanto, quando se considera as espécies que ocorreram concomitantemente nos três ambientes verificou-se que Baccharis elliptica, Calliandra sp., Cunila sp, Eremanthus erythropappus, Eremanthus incanus, Tibouchina candolleana e Trembleya cf. parviflora totalizaram juntas $21,87 \%$ da amostra total (Figura $1 \mathrm{e}$ Tabela 2). Por outro lado, as espécies com ocorrência registrada em apenas um dos ambientes amostrados (com um ou mais indivíduos em cada área) representaram $65,62 \%$ (21 espécies) de todas as espécies amostradas. Destas, dez $(31,25 \%)$ ocorreram em A1, sete $(21,87 \%)$ em A3 e quatro (12,50\%) em A2 (Figura 1).

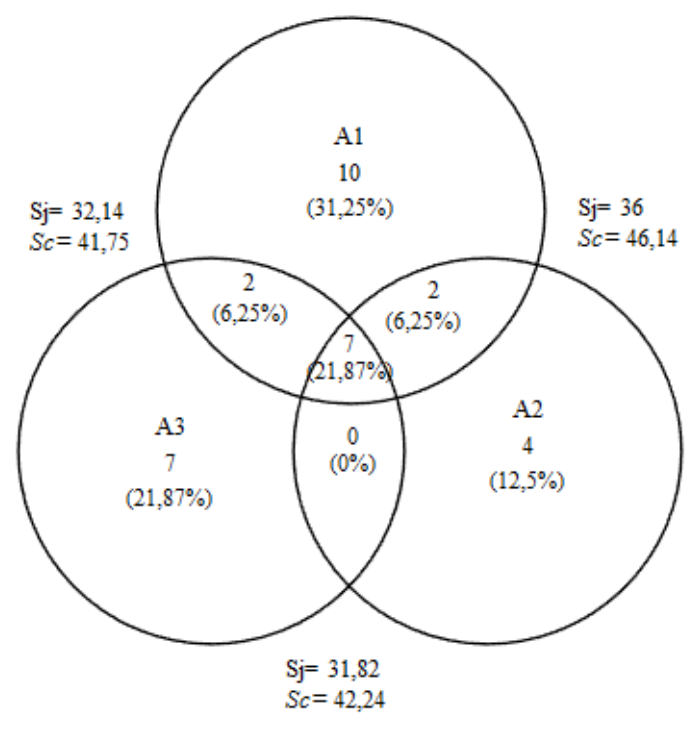

Figura 1: Diagramas de Venn produzidos a partir das espécies compartilhadas e exclusivas e seus respectivos índices de similaridade de Jacard ( $\mathrm{Sj}$ ) e Czekanowski (Sc) entre os três ambientes de uma cascalheira localizada no Parque Estadual do Biribiri, em Diamantina, MG. Onde: A1=Ambiente 1; $\mathrm{A} 2=$ Ambiente 2 e A3=Ambiente 3 . 
Quando se analisa a riqueza em espécies verifica-se considerável discrepância entre os ambientes (Tabela 3 ), onde A1 foi o mais rico (21 espécies), seguido por A3 (16), e A2 (13). A diferenciação entre as áreas é confirmada quando se compara a riqueza de espécies pelos estimadores 'jackknife' que indicaram riqueza potencial em espécies mais elevada para A1. Em contrapartida, A2 foi o ambiente que apresentou a menor riqueza dentre os demais amostrados.

Tabela 3. Variáveis estruturais amostradas em 20 parcelas $(5 \mathrm{~m} \times 5 \mathrm{~m})$ em três ambientes de uma cascalheira localizada no Parque Estadual do Biribiri, Diamantina, MG.

\begin{tabular}{lrrrc}
\hline Variáveis Estruturais & A1 & A2 & A3 & H \\
\hline Riqueza de espécies & 21 & 13 & 16 & - \\
Número de indivíduos & 844 & 270 & 497 & - \\
Shannon (H') & 2,074 a & 1,848 b & 1,680 c & $*$ \\
Pielou (J') & 0,681 & 0,721 & 0,606 & - \\
Estimador Jackknife; $1^{\circ}$ ordem & 28,6 & 15,9 & 23,6 & - \\
Estimador Jackknife; $2^{\circ}$ ordem & 34,1 & 16,8 & 29,9 & - \\
Densidade de espécies & $5,9 \pm 2,45$ a & $3,75+1,8$ b & $4,2 \pm 1,67$ a,b & $84833^{*}$ \\
Densidade de indivíduos & $42,2 \pm 43,66$ a & $13,5+9,94$ b & $24,85 \pm 27,2$ a,b & $70004^{*}$ \\
\hline
\end{tabular}

Onde: * significativo a 5\% de probabilidade pelo teste $\mathrm{t}$ de Hutcheson; $\mathrm{A} 1=$ ambiente $1 ; \mathrm{A} 2=$ Ambiente 2 e A3 = Ambiente $3 ; \mathrm{H}=$ teste $\mathrm{t}$ de Hutcheson. Os valores de densidade de espécies e indivíduos são médias \pm desvios padrão das 20 parcelas em cada ambiente.

Quando se utilizou as parcelas de cada ambiente como unidades de amostra da riqueza (Densidade de espécies), verificou-se pelo teste de Kruskal-Wallis a existência de diferenças entre as áreas (Tabela 3). Assim sendo, a densidade de espécies por parcela foi considerada significativamente diferente $(p<0,05)$ entre os três ambientes amostrados, sendo A1 considerado diferente de A2, porém ambos sendo semelhantes a A3.

A baixa riqueza observada pode ser decorrente dos níveis de degradação de cada ambiente, já que a permanência dos fatores de degradação nas áreas tornaria estes ambientes menos propícios à germinação e ao estabelecimento de uma ampla variedade de espécies (Harper et al., 2005). Além disso, tais impactos poderiam limitar a presença de animais, o que levaria à redução das chuvas de sementes oriundas de remanescentes adjacentes e restringiria o recrutamento de indivíduos (Benitez-Malvido, 1998). De fato, a presença permanente ou mesmo recente de alterações de habitat decorrentes das ações humanas contribuem para a degradação e perda de biodiversidade (Saunders et al., 1991; Gascon et al., 2000; Harper et al., 2005).

Tais hipóteses foram previstas pela teoria de distúrbios intermediários (Connell, 1978) a qual sugere que a diversidade e riqueza de espécies podem aumentar sob pequenos distúrbios, mas em contrapartida, a continuidade de distúrbios por longos períodos de tempo levariam algumas espécies a apresentarem baixas densidades, devido à ausência de condições para a colonização, ou seja, sob altas taxas de distúrbios, poucas espécies conseguem sobreviver o que acarreta tendências de monopolização do habitat.

Ao se analisar os valores dos índices de diversidade de Shannon (H') para cada ambiente verificou-se variação de 1,680 a 2,074 nats.indivíduo ${ }^{-1}$, onde $\mathrm{A} 3$ foi a área que apresentou o menor índice de diversidade e A1 a de maior valor.

A comparação entre índices de diversidade feita pelo teste- $t$ de Hutcheson mostrou que os três ambientes foram significativamente diferentes entre si (teste- $t$ de Hutcheson; $p<0,05$ ).

A Equabilidade de Pielou ( $\mathrm{J}$ ') variou entre 0,606 a 0,721 , com o maior valor foi amostrado em $\mathrm{A} 1$ e o menor em A3, semelhante ao obtido para H'. Os baixos valores de J' observados para os três ambientes investigados sugerem a existência de alta dominância ecológica de algumas espécies.

Possivelmente os locais estudados não apresentavam condições adequadas para o desenvolvimento de grande número de espécies, já que a maioria delas não suportaria 
condições limitantes, como elevada compactação e a consequente baixa disponibilidade hídrica do solo, dentre outras características apresentadas pelos ambientes. Além disso, valores de resistência mecânica à penetração entre 2,45 e 4,9 Mpa resulta em limitações para o crescimento e desenvolvimento adequado das plantas (Ribeiro et al., 2006; Oliveira et al., 2007). Assim, poucas espécies teriam capacidade para colonizar aqueles ambientes, o que gerou a elevada dominância ecológica, observada pelos baixos valores tanto do índice de Shannon como pela equabilidade de Pielou.

Observou-se que os ambientes estudados apresentavam flora consideravelmente distinta (Figura 1). Esta baixa similaridade foi observada pelos índices de Jaccard encontrados para os três ambientes, que se mostraram abaixo de 50\%. Segundo Gauch (1982), esse seria o valor mínimo utilizado para considerar áreas similares floristicamente. Isto indica que o número de espécies compartilhadas entre os ambientes é pequeno, o que é reforçado pela baixa porcentagem de espécies $(21,87 \%)$ registradas em comum a todos os ambientes (Figura 2). Quanto à similaridade estrutural (densidade), também foi considerada de baixa semelhança entre as áreas, em função do índice de Czekanowski em todos os ambientes ser inferior a 47\% (Figura 1).

Esta elevada distinção florística e consequentemente baixo compartilhamento de espécies entre os três locais estudados, provavelmente, deve ser resultante das diferenças ambientais ocasionadas pela distinta intensidade de degradação que os ambientes foram subordinados. Assim sendo, como os fatores ambientais são possivelmente diferentes, observado visualmente in situ, espécies distintas se adaptariam melhor a cada condição e, consequentemente, a cada ambiente. Outros estudos, também realizados em áreas de mineração, observaram que locais com diferentes históricos de uso podem apresentar grande variação na composição das espécies, gerando baixas taxas de similaridade entre áreas (Norman et al., 2006; Koch, 2007). Por esta razão, compreender os padrões sucessionais de áreas degradadas, especialmente pela atividade de mineração, pode auxiliar em programas de recuperação (Koch, 2007).

No conjunto total dos dados foram amostrados 1.611 indivíduos, sendo que A1 e A3 foram as áreas com maior densidade (844 e 497 indivíduos, respectivamente) enquanto que $\mathrm{A} 2$ foi o ambiente com a menor densidade (270). Quando os valores das densidades de indivíduos por parcelas foram comparados pelo teste de KruskalWallis foi verificada que esta é estatisticamente diferente entre as áreas, sendo pelo teste de Dunn, A1 diferente de A2, porém, estes foram considerados semelhantes a A3 (Tabela 3).

Quando os ambientes foram avaliados separadamente tanto para A1 como para A2, as cinco espécies mais abundantes foram: Baccharis elliptica (A1: 167 e A2: 52 indivíduos), Eremanthus erythropappus (A1: 153 e A2: 79), Cunila sp. (A1: 141 e A2: 54), Eremanthus incanus (A1: 140 e A2: 42) e Tibouchina candolleana (A1: 133 e A2: 20) que representaram respectivamente, $84 \%$ e $90 \%$ da amostragem destes ambientes. No entanto, quando analisado $\mathrm{A} 3$ as cinco espécies mais abundantes foram: B. elliptica (211), Cuphea campestris (112), Calliandra sp. (57), E. erythropappus (42), E. incanus (30) (Tabela 2) que juntas representaram mais de $91 \%$ da abundância neste ambiente. Estes resultados reforçam a existência de elevada dominância ecológica nas áreas estudadas.

Com relação aos valores do índice de regeneração (Tabela 2) verifica-se que as espécies com os maiores valores em A1 foram: B. elliptica $(17,95 \%)$, E. incanus $(17,12 \%)$, E. erythropappus $(15,50 \%)$, T. candolleana $(12,54 \%)$ e Cunila sp. (10,48\%) (Tabela 1), as quais juntas totalizaram $73,59 \%$ da amostragem. Já as espécies E. incanus $(25,30 \%)$, Cunila sp. (18\%), B. elliptica $(17,63 \%)$, E. erythropappus (14,45\%) e T. candolleana $(8,37 \%)$ foram as que apresentaram maior regeneração em A2 e estas juntas perfizeram um total de $83,75 \%$. No ambiente 3 , os maiores valores de cobertura foram encontrados para as espécies B. elliptica $(33,13 \%)$, C. campestris $(14,84 \%)$, E. erythropappus $(13,16 \%)$, Calliandra sp. $(11,09 \%)$ e $E$. incanus $(10,76 \%)$, as quais totalizaram $82,98 \%$ da regeneração.

Quando comparados os três ambientes, observa-se que apesar de ocorrem na mesma área e a uma distância relativamente curta, observaram-se variações tanto nos padrões florísticos quanto estruturais. Novamente, as distintas características ambientais podem ter sido as responsáveis por tais variações, o que sugere a necessidade de diagnósticos detalhados de locais a serem recuperados, pois dentro de uma mesma área, é possível encontrar ambientes com diferentes níveis de degradação que demandariam intervenções diferenciadas.

Assim sendo, como A1 apresenta o menor nível de degradação (solos menos compactados e sem processos erosivos, com terreno menos acidentado, dentre outras características) é possível que os propágulos que 
ali chegaram tenham encontrado condições menos restritivas à sua germinação e estabelecimento, favorecendo assim a maior riqueza apresentada por este ambiente, a qual foi confirmada pelos valores dos estimadores de Jackknife. Assim sendo, neste ambiente um número maior de espécies conseguiu se estabelecer e encontrar condições para o desenvolvimento desses indivíduos, resultando em menor dominância ecológica que os demais ambientes. Outro aspecto que pode ter favorecido a maior riqueza de $\mathrm{A} 1$ é a maior proximidade de um campo natural $(50 \mathrm{~m})$, que pode ter funcionado como fonte de propágulos.

A2 foi o ambiente que apresentou os menores valores nos parâmetros florísticos e estruturais testados. E, assim como em A1, o nível de degradação pode ter sido um dos responsáveis por tais resultados. Como este ambiente é mais degradado que o anterior, as condições ambientais disponíveis provavelmente não foram adequadas para o estabelecimento de muitas espécies, o que afetou a florística e a densidade.

A declividade do terreno em A2 também pode ter limitado o estabelecimento de indivíduos na área, afetando assim a densidade, pois em função do terreno acidentado, propágulos que por ventura tenham chegado neste ambiente podem ter sido carreados para outras porções da cascalheira devido às chuvas.

O ambiente 3, apesar de apresentar o maior nível de degradação, mostrou valores intermediários com relação à riqueza e densidade. Isso possivelmente se deve ao fato de A3 estar próximo a um candeal, o que favorece a disponibilidade de propágulos. O tempo em que este local se encontra em processo de regeneração também pode ter influenciado nessa riqueza diferenciada, pois como a extração de cascalho na área foi irregular e não se sabe ao certo quando foi encerrada, o tempo disponível

(A)

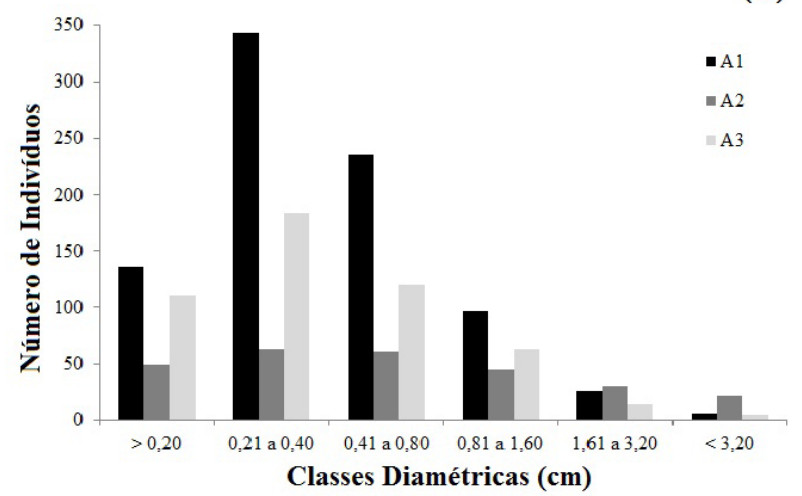

para essa recolonização pode ter sido maior neste que nos outros locais estudados, o que por consequência teria favorecido a chegada e estabelecimento de espécies.

No entanto, a relação entre número de espécies e a distribuição de indivíduos indicou que, apesar de A3 apresentar uma riqueza intermediária, a maioria destas espécies possui poucos indivíduos, ou seja, um número pequeno de espécies detém a maioria dos indivíduos, gerando assim dominância ecológica. Esta provavelmente está relacionada à limitação ambiental que este ambiente impõe, pois em virtude da degradação apresentada, mesmo que um número considerável de espécies se estabeleçam, seus indivíduos estarão limitados, possivelmente se desenvolvendo em porções menos restritivas do terreno, especialmente em relação à compactação.

Observou-se elevada concentração de indivíduos nas categorias intermediárias de classes diamétricas, nos três ambientes (Figura 2a). Este padrão, conhecido como unimodal, é caracterizado pela distribuição em forma de sino, apresentando um ponto com maior frequência (Umaña \& Alencar, 1998). As duas classes intermediárias $(0,21$ a 0,40 e 0,41 a $0,80 \mathrm{~cm})$ somadas representaram mais de $60 \%$ da amostragem em A1 eA3 e mais de $45 \%$ em A2. No entanto, o padrão de distribuição das alturas, para os três ambientes, apresentou distribuição do tipo $J$-invertido (Figura $2 b$ ), ou seja, com elevada concentração de indivíduos nas menores classes e redução acentuada em direção às maiores. As duas primeiras classes $(0,1$ a 0,8$)$ juntas detiveram mais de $78 \%$ dos indivíduos em três ambientes. Esses resultados demonstram que a comunidade foi formada por indivíduos de alturas reduzidas (menores que $1 \mathrm{~m}$ ), reforçando a hipótese de que os indivíduos amostrados eram jovens.

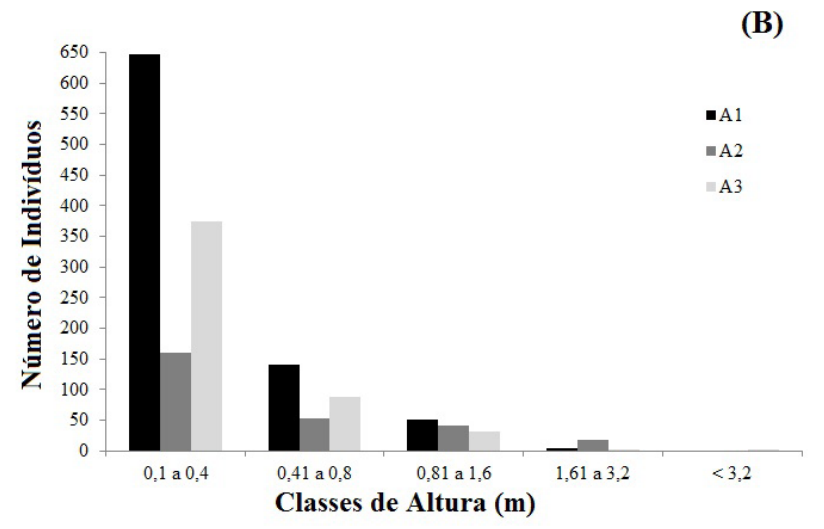

Figura 2: Distribuição em classes de diâmetro (A) e de altura (B) dos indivíduos amostrados em três ambientes de uma cascalheira localizada no Parque Estadual do Biribiri, Diamantina, MG. Onde: A1 = Ambiente 1; A2 = Ambiente 2 e A3 $=$ Ambiente 3 . 
As distribuições diamétricas e de alturas encontradas apresentaram uma inversão nos padrões normalmente encontrados em estudos de regeneração natural. Em geral, a literatura indica curva unimodal para a variável altura e distribuição $J$-invertido para diâmetro (Nappo et al., 2005; Narvaes et al., 2005; Paula et al., 2009). A distribuição em altura, que foi caracterizada pela curva em $J$-invertido, pode ser atribuída à existência de muitos indivíduos jovens. Segundo Gama et al. (2002) e Redin et al. (2011) a diminuição do número de plantas com o aumento das classes de tamanho é um padrão esperado em estudos de regeneração natural e sugere que a área se encontra em fase de construção, o que pode ser um indício de que a área em questão também estaria nesse estágio sucessional. No entanto, não se pode descartar a permanência de ações antrópicas que poderiam restringir o desenvolvimento de indivíduos nas maiores classes.

De acordo com Péllico-Netto et al. (1997) diferenças drásticas entre classes de altura, como a observada entre a primeira e segunda classe, podem sugerir influência de ação antrópica no desenvolvimento da regeneração natural. Esta mesma explicação pode estar relacionada à distribuição do tipo unimodal apresentada pela variável diâmetro. Port, limitações dos ambientes dificultariam tanto o ingresso de indivíduos nas classes menores como o desenvolvimento destes em classes superiores. Assim sendo, a maioria dos indivíduos permaneceriam nos intervalos intermediários. De fato, Paula et al. (2009) estudando a regeneração natural em área de Cerrado no Brasil Central, também observaram forte relação entre a baixa representatividade de indivíduos de menor porte com a influência de antropização na distribuição diamétrica da comunidade vegetal.

Segundo Husch et al. (1982), a distribuição dos indivíduos ao longo de classes de diâmetro e altura de uma comunidade, natural ou antropizada, varia durante os anos devido a fatores que alteram padrões de desenvolvimento, como mortalidade, ou mesmo corte dos indivíduos do local. Por esta razão, a forma mais apropriada para compreender a distribuição de indivíduos em classes em um ambiente é monitorá-lo periodicamente e por longos períodos (Schaaf et al., 2006; Redin et al., 2011).

Das 32 espécies amostradas no levantamento e analisadas como indicadoras (Tabela 4), dez (31,25\% da amostragem total) apresentaram preferência significativa por um dos três ambientes estudados, sendo seis (18,75\%) para A1 (T. candolleana, Hirtella glandulosa, E. erythropappus, Erythroxylum cuneifolium, E. incanus e H. crinita), três $(9,37 \% \%)$ para A3 (B. elliptica, Calliandra sp. e C. campestris) e apenas uma espécie $(3,12 \%)$ foi considerada indicadora para A2 (Solanum cf grandiflorum).

Tabela 4: Análise de espécie indicadoras (ISA) realizada com base na lista e na abundância das espécies dos três ambientes da cascalheira localizada no Parque Estadual do Biribiri, Diamantina, MG.

\begin{tabular}{|c|c|c|c|c|c|c|c|}
\hline \multirow{2}{*}{$\begin{array}{l}\text { Áreas } \\
\quad \text { Espécies }\end{array}$} & \multirow{2}{*}{ VIO } & \multicolumn{3}{|c|}{ VIE } & \multicolumn{3}{|c|}{ Abundância (\%) } \\
\hline & & Média & $s$ & $p$ & A1 & A2 & $\mathbf{A 3}$ \\
\hline \multicolumn{8}{|l|}{ Ambiente 1} \\
\hline Tibouchina candolleana & 47,4 & 26,2 & 7,32 & $* *$ & 82 & 11 & 7 \\
\hline Hirtella glandulosa & 21,1 & 7,5 & 3,55 & $* * *$ & 100 & 0 & 0 \\
\hline Eremanthus incanus & 53,4 & 31,9 & 5,29 & $* *$ & 63 & 18 & 18 \\
\hline Eremanthus erythropappus & 60,5 & 36,3 & 5,70 & $* * *$ & 60 & 28 & 11 \\
\hline Erythroxylum cuneifolium & 36,8 & 10,3 & 4,53 & $* * *$ & 100 & 0 & 0 \\
\hline Hyptis crinita & 57,3 & 17,0 & 5,09 & $* * *$ & 84 & 16 & 0 \\
\hline \multicolumn{8}{|l|}{ Ambiente 2} \\
\hline Solanum cf. grandiflorum & 23,8 & 8,0 & 3,80 & $* *$ & 0 & 100 & 0 \\
\hline \multicolumn{8}{|l|}{ Ambiente 3} \\
\hline Baccharis elliptica & 48,4 & 36,8 & 5,33 & $*$ & 39 & 12 & 48 \\
\hline $\begin{array}{l}\text { Calliandra sp. } \\
\text { Cuphea campestris }\end{array}$ & $\begin{array}{l}41,4 \\
30,0\end{array}$ & $\begin{array}{l}14,4 \\
10,4\end{array}$ & $\begin{array}{l}4,96 \\
4,69\end{array}$ & $\begin{array}{l}* * \\
* * *\end{array}$ & $\begin{array}{l}3 \\
0\end{array}$ & $\begin{array}{l}5 \\
0\end{array}$ & $\begin{array}{c}92 \\
100\end{array}$ \\
\hline
\end{tabular}

Onde: *,** e*** significativo à $5 \%, 1 \%$ e $0,1 \%$ de probabilidade, respectivamente; VIO $=$ valor indicador observado; VIE $=$ valor indicador esperado; $\mathrm{s}=$ desvio padrão; $\mathrm{p}=$ significância. 
Poucas espécies foram consideradas indicadoras dos ambientes. Isto possivelmente está relacionado à baixa riqueza florística apresentada pelos mesmos. Essa relação entre o número de espécies indicadoras e a riqueza também pode ser observada quando se considera cada ambiente separadamente. Assim sendo, A1 que foi o local com maior densidade de espécies apresentou maior número de espécies indicadoras, em contrapartida, A2 que foi o ambiente de menor riqueza apresentou apenas uma espécie indicadora.

Em muitos casos houve uma confirmação das espécies indicadoras de cada ambiente com as espécies de maiores valores de regeneração nas áreas, indicando que estas exercem bastante importância nos ambientes. Desta forma, três das cinco espécies preditoras de A1 estavam entre as cinco espécies de maiores valores de regeneração natural. Já no caso de A3 as três espécies selecionadas como indicadoras apresentavam elevados valores de densidades e frequências neste ambiente. No entanto, esse padrão não foi observado para $\mathrm{A} 2$, pois a única espécie indicadora deste ambiente (Solanum cf grandiflorum) não se encontra entre as de maiores valores de regeneração natural.

Ao se analisar os ambientes separadamente e suas respectivas espécies preditoras, verificou-se que a degradação de cada local exerceu peso na seleção destas espécies. Desta forma, A1 que era o ambiente menos degradado, apresentou grande contribuição de espécies de porte arbóreo, com três das cinco espécies indicadoras sendo árvores. Portanto, como este ambiente apresentou histórico de uso mais leve, as condições ambientais eram propícias ao estabelecimento de espécies de porte arbóreo como T. candolleana, H. glandulosa, E. incanus a ponto destas serem selecionadas como indicadoras.

No caso de A2, ambiente com nível intermediário de degradação, observou-se apenas Solanum cf grandiflorum como espécie indicadora. Esta apresenta porte mais arbustivo e é comumente encontrada colonizando ambientes degradados na região do presente estudo. O gênero Solanum tem sido frequentemente apontado como abundante em áreas antropizadas (Guarana et al., 2011) e esta ocorrência se deve à sua elevada capacidade de colonizar ambientes onde as condições hídricas e edáficas não são favoráveis ao desenvolvimento de outras espécies (Elias et al., 2003), principalmente de espécies arbóreas. No caso de A3, ambiente com maior intensidade de antropização, foram selecionadas como indicadoras apenas espécies tipicamente arbustivas, que apresentam porte reduzido e consequentemente com menores exigências edafo-climáticas.

Desta forma, com base nas especificidades destas espécies às condições ambientais de cada área e ao fato destas serem consideradas importantes para o comportamento estrutural destes ambientes, estas podem ser consideradas com potencial para uso em projetos de restauração em áreas de mineração na região com condições semelhantes à cascalheira estudada.

\section{Conclusões}

Os ambientes estudados apresentaram diferenciações florísticas e estruturais e estas, provavelmente, se devem às variações do ambiente impostas pelas diferentes intensidades de degradação dos mesmos.

Os impactos advindos da atividade de mineração continuam exercendo forte influência na capacidade de retomada da sucessão nestes ambientes, sendo necessária a interferência antrópica por meio de projetos de restauração.

Na recuperação destes ambientes devem-se priorizar as espécies indicadoras encontradas devido à importância ecológica e estrutural que estas poderiam desenvolver nestes locais.

\section{Referências}

ANAND, M.; DESROCHERS, R. E. Quantification of restoration success using complex systems concepts and models. Conservation Biology, Boston, v. 12, n. 1, p. 117-123, 2004.

ANGIOSPERM PHYLOGENY GROUP. An update of the Angiosperm Phylogeny Group classification for the orders and families of flowering plants: APG III. Botanical Journal of the Linnean Society, London, v. 161, p. 105-121, 2009.

BENITEZ-MALVIDO, J. Impact of forest fragmentation on seedling abundance in a tropical rain forest. Conservation Biology, Boston, v 12, p. 380-389, 1998.

BENTES-GAMA, M. M.; PEREIRA, N. S.; CAPELASSO, P. H. S.; SALMAN, A. K. D.; VIEIRA, A. H. Espécies arbóreas nativas com potencial para recuperação de paisagens alteradas em Rondônia. Porto Velho: Embrapa Rondônia, 2008. 41 p. (Embrapa Rondônia. Documentos, 128).

BORSAGLI, A. A influência dos caminhos e estradas na consolidação da malha urbana de Diamantina. Webartigos. 2010. Disponível em: <http://www.webartigos.com/articles/34756/1/a-influenciados-caminhos-e-estradas-na-consolidacao-da-malha-urbana-dediamantina-minas-gerais/paginal.html> . Acesso em: 29 ago. 2011.

BROWER, J. E.; ZAR, J. H. Field \& laboratory methods for general ecology. Boston: Brown Publishers, 1984. 226 p. 
CONNELL, J. H. Diversity in tropical rain forest and coral reefs. Science, New York, v. 199, p. 1302-1310, 1978.

COOKE, J. A.; JOHNSON, M. S. Ecological restoration of land with particular reference to the mining of metals and industrial minerals: a review of theory and practice. Environmental Review, Ottawa, v. 10, p. $41-71,2002$.

DUFRÊNE, M.; LEGENDRE, P. Species assemblages and indicator species: the need for flexible asymmetrical approach. Ecological Monographs, v. 67, n. 3, p. 345-366, 1997.

ELIAS, S. R. M.; ASSIS, R. M.; STACCIARINI-SERAPHIN, E.; REZENDE, M. H. Anatomia foliar em plantas jovens de Solanum lycocarpum A.St.-Hil. (Solanaceae). Revista Brasileira de Botânica, São Paulo, v. 26, n. 2, p. 169-174, 2003.

EMPRESA BRASILEIRA DE PESQUISA AGROPECUÁRIA - EMBRAPA. Centro Nacional de Pesquisa de Solos. Sistema Brasileiro de Classificação de Solos. Rio de Janeiro: Embrapa Solos, 2006. 306p.

FERREIRA, W. C.; BOTELHO, S. A.; DAVIDE, A. C.; FARIA, J. M. R. Avaliação do crescimento do estrato arbóreo de área degradada revegetada à margem do Rio Grande, na usina hidrelétrica de Camargos, MG. Revista Árvore, Viçosa, MG, v. 31, n. 1, p. 177 185, 2007.

GAMA, J. R. V.; BOTELHO, S. A.; BenteS-GAMA, M. M. Composição florística e Estrutura da regeneração natural de floresta secundária de Várzea Baixa no Estuário Amazônico. Revista Árvore, Viçosa, MG, v. 26, n. 5, p. 559-566, 2002.

GASCON, C.; WILLIAMSON, G. B.; FONSECA, G. A. B. Receding forest edges and vanishing reserves. Science, New York, v. 288, p. 1356-1358, 2000.

GAUCH, H. G. Multivariate analysis in community ecology. Cambridge: Cambridge University, 1982. 298 p.

GOMES, A. S.; PAlmA, J. J. C.; SIlVA, C. G. Causas e conseqüências do impacto ambiental da exploração dos recursos minerais marinhos. Brazilian Journal of Geophysics, Rio de Janeiro, v. 18, n. 3, p. 447-454, 2000.

GUARANA, E. L. S. RIET-CORREA, F.; MENDONÇA, C. L.; MEDEIROS, R. M. T.; COSTA, N. A.; AFONSO, J. A. B. Intoxicação por Solanum paniculatum (Solanaceae) em bovinos. Pesquisa Veterinária Brasileira, Rio de Janeiro, v. 31, n. 1, p. 59-64, 2011.

HARPER, K. A.; MACDONALD, S. E.; BURTON, P. J.; CHEN, J.; BROSOFSKE, K. D.; SAUNDERS, S. C.; EUSKIRCHEN, E. S.; ROBERTS, D.; JAITEH, M. S.; ESSEN, P. Edge influence on forest structure and composition in fragmented landscapes. Conservation Biology, Boston, v. 3, p. 768-782, 2005.

HELTSCHE, J. F.; FORRESTER, N. E. Estimating species richness using the jackknife procedure. Biometrics, Washington, US, v. 39, p. 1-12, 1983.

HUSCH, B.; MILLER, C. I.; BEERS, T. W. Forest mensuration. New York: John Wiley, 1982. 402 p.

KAMINO, L. H. Y.; OLIVEIRA-FILHO, A. T.; STEHMANN, J. R. Relações florísticas entre as fitofisionomias florestais da Cadeia do Espinhaço, Brasil. Megadiversidade, Belo Horizonte, v. 4, n. 1-2, p. 38-77, 2008.
KENT, M.; COKER, P. Vegetation description analyses. London: Behaven, 1992. 363 p.

KLEIN, A. S.; CITADINI-ZANETTE, V.; LOPES, R. P.; SANTOS, R. Regeneração natural em área degradada pela mineração de carvão em Santa Catarina, Brasil. Revista Escola de Minas, Ouro Preto, v. 62, n. 3, p. 297-304, 2009.

$\mathrm{KOCH}, \mathrm{J}$. M. Alcoa's mining and restoration process in south Western Australia. Restoration Ecology, Malden, v. 15, p. S11-S16, 2007.

MCCUNE, B.; MEFFORD, M. J. PC-ORD: multivariate analysis of ecological data: version 6. Gleneden Beach: MjM Software, Oregon, 2011.

MUELLER-DOMBOIS, D.; ELLENBERG, H. Aims and methods of vegetation ecology. Caldwell: The Blackburn, 2002. 548 p.

NAPPO, M. E.; FONTES, M. A. L.; OLIVEIRAFILHO, A. T. Regeneração natural em sub-bosque de povoamentos homogêneos de Mimosa scabrella Bentham, implantados em áreas mineradas, em Poços de Caldas, Minas Gerais. Revista Árvore, Viçosa, MG, v. 24, n. 3, p. 297-307, 2000.

NAPPO, M. E.; GRIFFITH, J. J.; MARTINS, S. V.; MARCO JÚNIOR, P.; SOUZA, A.L.; OLIVEIRA-FILHO, A. T. Dinâmica da estrutura diamétrica da regeneração natural de espécies arbóreas e arbustivas no sub-bosque de povoamento puro de Mimosa scabrella Bentham, em área minerada, em Poços de Caldas, MG. Revista Árvore, Viçosa, MG, v. 29, n. 1, p. 35-46, 2005.

NARVAES, I. S.; BRENA, D. A.; LONGHI, S. J. Estrutura da regeneração natural em floresta ombrófila mista na Floresta Nacional de São Francisco de Paula, RS. Ciência Florestal, Santa Maria, RS, v. 15 , n. 4 , p. 331-342, 2005.

NEVES, S. C.; ALMEIDA-ABREU, P. A.; FRAGA, L. M. S. Fisiografia. In: SILVA, A. C.; PEDREIRA, L. C. V. S. F.; ALMEIDAABREU, P. A. (Ed.). Serra do Espinhaço Meridional: Paisagem e ambiente. Belo Horizonte: O Lutador, 2005. p.137-147.

NORMAN, M. A.; KOCH, J. M.; GRANT, C. D.; MORALD, T. K.; WARD, S. C. Vegetation succession after bauxite mining in Western Australia. Restoration Ecology, Malden, v. 14, n. 2, p. 278-288, 2006.

OLIVEIRA, G. C.; SEVERIANO, E. C.; MELLO, C.R. Dinâmica da resistência à penetração de um Latossolo Vermelho da Microrregião de Goiânia. Revista Brasileira de Engenharia Agrícola e Ambiental, Campina Grande, v. 1, p. 265-270, 2007.

PARROTTA, J. A.; KNOWLES, O. H.; WUNDERLE, J. M. Development of floristic diversity in 10 -year-old restoration forests on a bauxite mined site in Amazonia. Forest Ecology and Management, Amsterdam, v. 99, p. 21-42, 1997.

PAULA, J. E.; IMAÑA-ENCINAS, J.; SANTANA, O. A.; RIBEIRO, G. S.; IMAÑA, C. R. Levantamento florístico e sua distribuição diamétrica da vegetação de um cerrado sensu stricto e de um fragmento de floresta de galeria no ribeirão Dois Irmãos na APA de Cafuringa, DF, Brasil. Revista Biotemas, Florianópolis, v. 22, n. 3, p. 35-46, 2009.

PÉLLICO NETTO, S.; BRENA, D. A. Inventário florestal. Curitiba, 1997. $316 \mathrm{p}$. 
REDIN, C. G.; LONGHI, R. V.; WATZLAWICK, L. F.; LONGHI, S. J. Composição florística e estrutura da regeneração natural do Parque Estadual do Espinilho, RS. Ciência Rural, Santa Maria, RS, v. 41, n. 7, p. 1195-1201, 2011.

RIBEIRO, A. I.; LONGO, R. M.; TEIXEIRA FILHO, A.; MELO, W. J. Diagnóstico de uma área compactada por atividade mineraria, na floresta amazônica, empregando métodos geoestatísticos à variável resistência mecânica à penetração do solo. Acta Amazonica, Manaus, v. 36, n. 1, p. 83-90, 2006.

SANTOS, H. G. dos; JACOMINE, P. K. T.; ANJOS, L. H. C. dos; OLIVEIRA, V. A. de; OLIVEIRA, J. B. de; COELHO, M. R.; LUMBRERAS, J. F.; CUNHA, T. J. F. (Ed.). Sistema brasileiro de classificação de solos. 2. ed. Rio de Janeiro: Embrapa Solos, 2006. 306 p.

SAUNDERS, D. A.; HOBBS, R. J.; MARGULES, C. R. Biological consequences of ecosystem fragmentation: a review. Conservation Biology, Boston, v. 5, p. 18-32, 1991.
SCHAAF, L. B.; FIGUEIREDO-FILHO, A.; GALVÃO, F.; SANQUETTA, C. R.; LONGHI, S. J. Modificações florísticoestruturais de um remanescente de floresta Ombrófila Mista Montana no período entre 1979 e 2000. Ciência Florestal, Santa Maria, RS, v. 16, n. 3, p. 271-291, 2006.

SILVA, L. C. R.; CORRÊA R. S. Sobrevivência e crescimento de seis espécies arbóreas submetidas a quatro tratamentos em área minerada no Cerrado. Revista Árvore, Viçosa, MG, v. 32, n. 4, p. 731-740, 2008

UMAÑA, C. L. A.; ALENCAR, J. C. Distribuições diamétricas da floresta tropical úmida em uma área no Município de Itacoatiara AM. Acta Amazonica, Manaus, v. 28, p. 167-190, 1998.

ZAR, J. H. Biostatistical analysis. 3rd ed. New Jersey: PrenticeHall, 1996. 662 p. 